\title{
Microspore Abortion and Abnormal Tapetal Degeneration in BNS Thermo-sensitive Male Sterile Wheat Lines
}

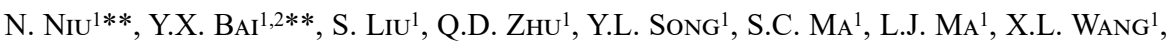 \\ G.S. ZHANG ${ }^{1 *}$ and J.W. WANG ${ }^{1 *}$ \\ ${ }^{1}$ College of Agronomy, Northwest A \& F University / Wheat Breeding Engineering Research Center, Ministry \\ of Education/Key Laboratory of Crop Heterosis of Shaanxi Province, Yangling, 712100, Shaanxi, China \\ ${ }^{2}$ Qinghai University/Qinghai Academy of Agricultural and Forestry Sciences/Qinghai Key Laboratory \\ of Hulless Barley Genetics and Breeding, Xining 810016, Qinghai Province, China
}

(Received 11 December 2018; Accepted 26 March 2019;

Communicated by S.P. Baenziger)

\begin{abstract}
Studies of the pollen abortion mechanism in thermo-sensitive male sterile lines may provide a strong foundation for breeding hybrid wheat and establishing a theoretical basis for marker-assisted selection. To investigate the cause of pollen abortion in Bainong thermo sensitive male sterile (BNS) lines, we analyzed the properties of pollen grains, changes in the tapetum and microspores in different anther developmental stages, and the distribution and deposition of nutrient substances in microspores. We found that tapetum degraded in the early uninucleate stage in sterile BNS (S-BNS), which was earlier than that of fertile BNS (F-BNS) tapetum. Large amounts of insoluble polysaccharides, lipids, and proteins were deposited until the trinucleate pollen stage in the nutritive cells in F-BNS. At the binucleate stage, the vacuoles disappeared and pollen inclusion increased gradually. At the trinucleate stage, these nutrients would help pollen grains mature and participate in fertilization normally. Therefore, early degradation of the tapetum, which inhibits normal microspore development, and the limited content of nutrient substances in pollen may be the main factors responsible for male sterility in BNS lines.
\end{abstract}

Keywords: pollen abortion, microspore development, nutrient, tapetum degradation, male sterility, wheat

Abbreviations: BNS, Bainong thermo-sensitive male sterile; F-BNS, Fertile-BNS; S-BNS, Sterile-BNS; IOD, Integrated optical density

\section{Introduction}

Breeding high-yielding cultivars has been achieved in rice (Oryza sativa L.), corn (Zea mays L.), and rape (Brassica napus L.) using heterosis (Karim et al. 2014), but it has not been achieved in wheat. Photo-thermo-sensitive male sterility is considered to be among the most promising method for utilizing heterosis in wheat because of its many advan-

\footnotetext{
*Corresponding author; E-mails: zhanggsh58@aliyun.com; wjw@nwsuaf.edu.cn

**These authors contributed equally to this work.
} 
tages, including its simple genetic basis, good performance in terms of sterility and restoration, and the capacity to easily produce excellent hybrid combinations (Ru et al. 2015). At present, the mechanism responsible for the changes in fertility in photo-thermo-sensitive lines is poorly understood, thereby hindering the development of hybrid wheat breeding. This study explored the relationship between nutrient metabolism in the tapetum and microspore abortion in BNS male sterile wheat based on cytological analyses.

The tapetum is closely associated with the developing gametophytes and it is the direct source of nutrients that contribute to pollen development (Regan and Moffatt 1990; Datta et al. 2001; Xie et al. 2005). The timing of tapetal programmed cell death plays an important role in the structure of the microspore and energy use (Endo et al. 2009). Many previous studies have shown that abnormal tapetal programmed cell death, either early or late, leads to pollen abortion (Parish and Li 2010; Zhang and Yang 2014). In rice, PERSISTENT TAPETAL CELL 1 (PTC1) encodes a zinc-finger protein that determines a switch for apoptosis and necrosis in tapetal cells, which is evident as delayed degradation and abnormal pollen wall formation. This gene has key roles in the regulation of tapetal development and pollen formation ( $\mathrm{Li}$ et al. 2011). TDR INTERACTING PROTEIN2 (TIP2) is a basic helix-loop-helix transcription factor in rice, which helps to control the division and differentiation of anther wall layers during early anther development. In tip2 mutants, the anther layers differentiate normally, whereas the middle layer and tapetum do not degrade normally during the later stages of pollen development. The growth of the microspore mother cells stops, thereby resulting in sterility (Fu et al. 2014).

The Bainong thermo-sensitive male sterile (BNS) wheat lines developed by the Henan Institute of Science and Technology, Xinxiang City, China have stable characteristics (Li et al. 2009; Zhang et al. 2010). The critical temperature for converion is $11.4{ }^{\circ} \mathrm{C}$. It showed complete male sterile when the temperature is below $11.4{ }^{\circ} \mathrm{C}$, and fertility when the temperature is above $11.4^{\circ} \mathrm{C}$. where abortion occurs during the period between stamen primordia differentiation and stamen differentiation. Male sterility in BNS lines is controlled by two pairs of major genes and multiple pairs of minor genes, as shown by the existence of gene-cytoplasm interactions (Zhang et al. 2013). Using cytochemical methods, Li et al. (2013) showed that the central vacuole in the nutritive cells of sterile pollen does not disappear and that cytoplasmic inclusion does not increase continuously during the binucleate stage. The disruption of starch deposition also leads to pollen abortion. However, the counterstaining technique used in the study by Li et al. (2013) did not allow lipid and protein deposition to be studied.

The objective of this study was to investigate the microspore abortion mechanism in BNS wheat lines by analyzing the properties of pollen grains, changes in the tapetum and microspores during different anther developmental stages, and the distributions and deposition of polysaccharides, lipids, and proteins in microspores. Our results provide a better understanding of the functional complementary between tapetal degeneration and substrate metabolism in anthers. 


\section{Materials and Methods}

\section{Experimental materials}

The plant materials used in this study comprised two BNS wheat lines, i.e. sterile (S)-BNS and fertile (F)-BNS, which were planted on October 1 and November 16, 2013, respectively, at the experimental station of Northwest A\&F University, Yangling, Shaanxi Province, China.

\section{Microscopic study of microspores}

In late April 2014, 30 fresh anthers from 10 middle florets of the different spikes in sterile line (planted on October 1, 2013) and fertility line (planted on November 16, 2013), respectively, when the anthers developed to the stage of binucleate or trinucleate by identifing the condition of the karyotypes and the vitality of the pollen grains on microscope.

\section{Sampling and distribution}

One anther from each floret was examined microscopically using the acetocarmine squash technique in order to identify the microspore development stage. Anthers were also fixed in Carnoy's solution to evaluate the pollen viability. In addition, anthers were crushed, stained with $1 \%(\mathrm{v} / \mathrm{v}) \mathrm{KI}-\mathrm{I}_{2}$, and examined using an light microscope (Olympus BX51, Japan. To determine karyotypes, pollen grains were stained with $1 \mu \mathrm{g} \mathrm{mL}^{-1} 4^{\prime}, 6$-diamidino-2-phenylindole (DAPI) and observed under ultraviolet light.

\section{Distribution of nutrients in anthers}

From April to May 2014, fresh anthers were collected and divided into two groups. One group was used for microscopic analysis to identify the microspore developmental stage. The anthers in the second group were embedded in Epon812 resin and sliced into $1 \mu \mathrm{m}$ thick sections with a Leica Em UC6 Ultramicrotome (Leica Microsystems, Germany). Sections of fertile and sterile anthers at different developmental stages were stained using periodic acid-Schiff (PAS) reagent, Sudan Black B (Ariizumi et al. 2004), and Coomassie Blue $\mathrm{R}-250$ (Hu and $\mathrm{Xu}$ 1990) to detect cytochemical changes that might occur during the distribution of insoluble polysaccharides (red), lipids (black), and proteins (blue), respectively.

\section{Data processing and analysis}

Four anther sections from each developmental stage were observed using an Olympus BX51 microscope equipped with the cellSens Entry package for digital imaging and documentation. The integrated optical density (IOD) was identified according to Ahern's method (Ahern 1998). The procedure was repeated six times at each developmental stage to decrease the operational error. All statistical analyses were performed using SPSS 19.0 (IBM, USA). 


\section{Results}

\section{Properties of pollen grains in BNS lines}

Microscopic analyses showed that the pollen grains in F-BNS were approximately spherical shaped and the sperm nuclei were torpedo shaped (Fig. 1a). The mature pollen grains from F-BNS appeared dark blue when stained with $\mathrm{I}_{2}$-KI (Fig. 1b). By contrast, the pollen grains from S-BNS were irregular in shape, were shrunken and dry, and their development was relatively slow and it stopped at the akaryote or uninucleate stages (Fig. 1c). The mature pollen grains from S-BNS were yellow or light brown when stained with $\mathrm{I}_{2}$-KI (Fig. 1d). These results demonstrate that there was a direct relationship between pollen abortion and abnormal nuclear division.
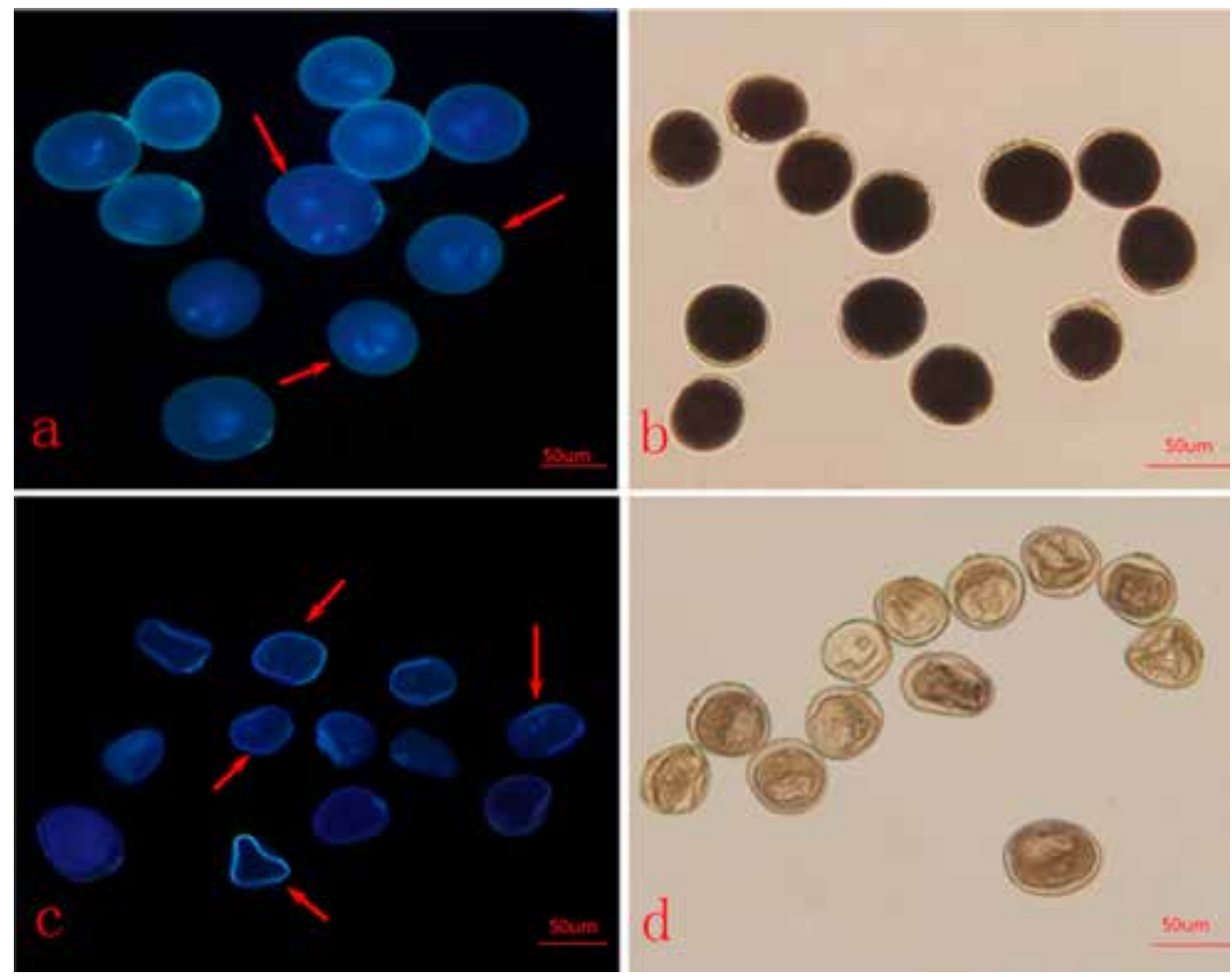

Figure 1. Cytological characteristics of pollen development in sterile-BNS (S-BNS) and fertile-BNS (F-BNS) in the trinucleate stage. (a) F-BNS pollen stained by DAPI; (b) F-BNS pollen stained by $1 \% \mathrm{I}_{2}-\mathrm{KI}$; (c) S-BNS pollen stained by DAPI; (d) S-BNS pollen stained by $\mathrm{I}_{2}-\mathrm{KI}$. Nuclei are indicated by arrows in S-BNS and F-BNS microspores. $\mathrm{Bar}=50 \mu \mathrm{m}$

\section{Changes in the tapetum and microspores in different anther development stages}

From the tetrad stage to the late uninucleate stage, the tapetum cells in microspores started to shrink in both F-BNS and S-BNS. Tapetal cells were identified from the binucleate 

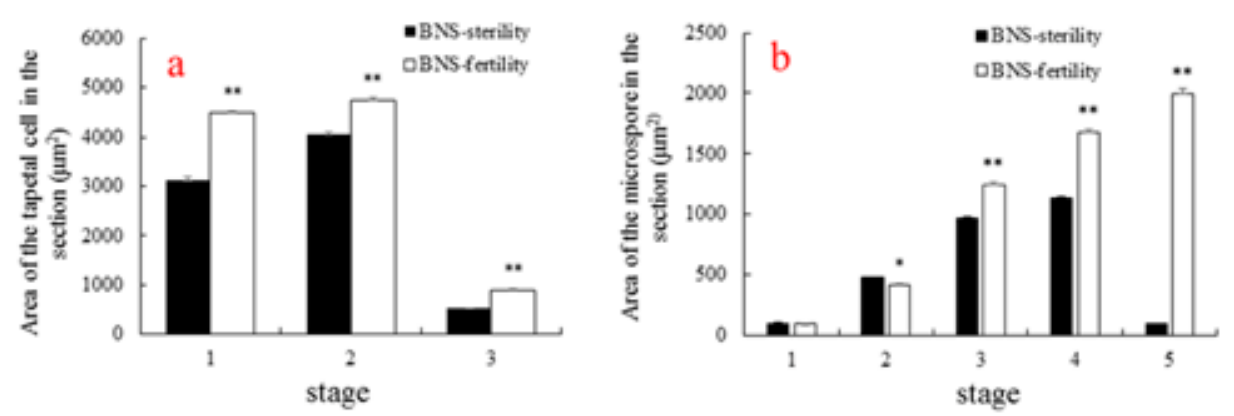

Figure 2. Section area $\left(\mu \mathrm{m}^{2}\right)$ of tapetal cells and microspores in different stages. (a) Tapetal cell section area; (b) Microspore section area. 1, Tetrad stage; 2, early uninucleate stage; 3 , late uninucleate stage; 4 , binucleate stage; 5 , trinucleate stage; ${ }^{*} p<0.05,{ }^{* *} p<0.01$

stage to the trinucleate stage, thereby indicating total tapetal degradation prior to the binucleate stage (Fig. 2a). In S-BNS, the tapetum decreased in size significantly faster at every developmental stage $(p<0.01)$ compared with those in F-BNS (Fig. 2a). During the tetrad stage, the microspores in S-BNS did not differ significantly from those in F-BNS (Fig. 2b). From the early uninucleate stage to the binucleate stage, the size of the microspores continued to increase in both F-BNS and S-BNS, but the microspores in F-BNS increased in size significantly faster $(p<0.01)$ than those in S-BNS (Fig. 2b). In the trinucleate stage, the microspores in F-BNS continued to increase in size, whereas those in S-BNS were shrunken and deformed (Fig. 2b).

\section{Distribution and deposition of polysaccharides in microspores}

Analysis based on IOD detected an increasing trend in the polysaccharide content of S-BNS microspores from the tetrad stage to the early uninucleate stage, but with a dramatic decrease in the late uninucleate stage (Fig. 3a-c), whereas the decrease was continuous from the tetrad stage until the late uninucleate stage in F-BNS microspores (Fig. $3 \mathrm{f}-\mathrm{h}$ ). Comparisons of the IOD values indicated that the polysaccharide content of S-BNS tapetum was significantly lower $(p<0.01)$ than that of F-BNS tapetum (Fig. 3k). In developmental stage, except for the uninucleate stage when the polysaccharide content of S-BNS microspores was significantly higher $(p<0.05)$ than that of F-BNS microspores, the IOD values of the polysaccharide content of S-BNS microspores other stage were lower than that of F-BNS microspores (Fig. 31). These results show that insoluble polysaccharides were deposited in F-BNS microspores from the tetrad stage until the late uninucleate stage and they were probably used for their further development.

\section{Distribution and deposition of proteins in microspores}

The IOD values showed that the protein contents of S-BNS tapetum started to decrease in the early uninucleate stage, which was earlier than that in F-BNS tapetum (Fig. $4 a-j)$. The 

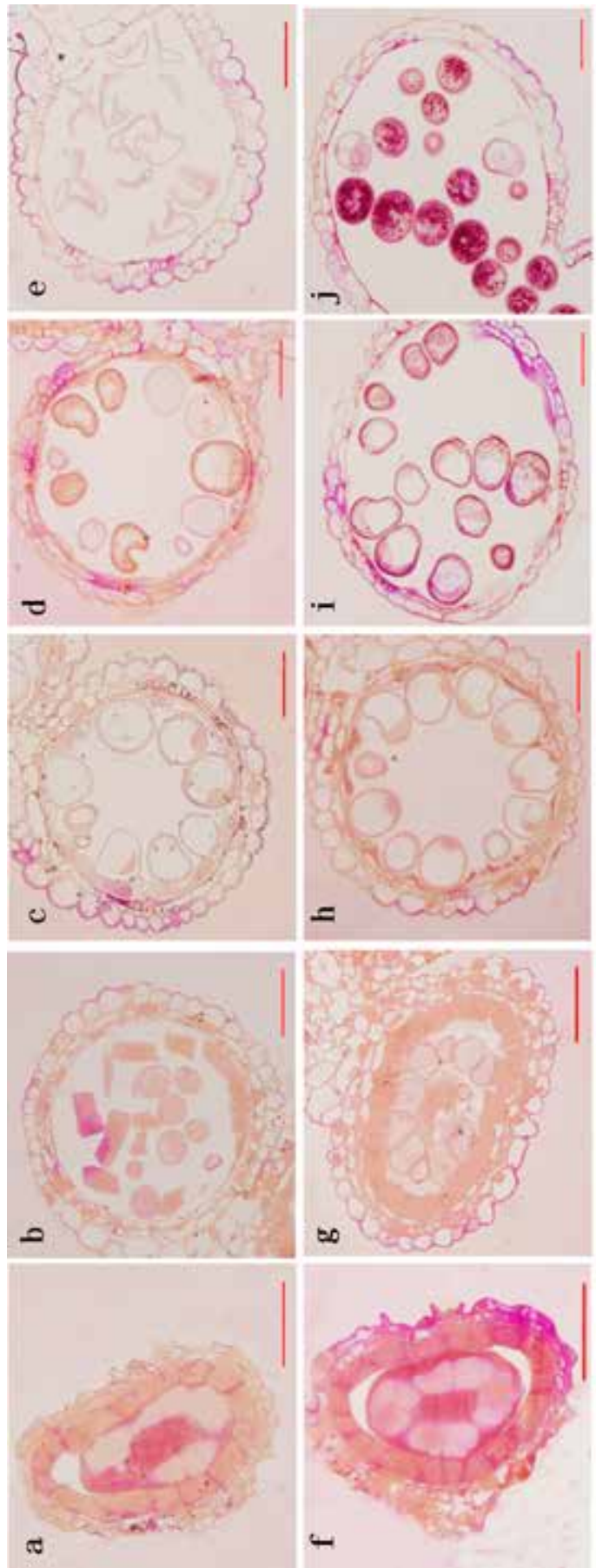

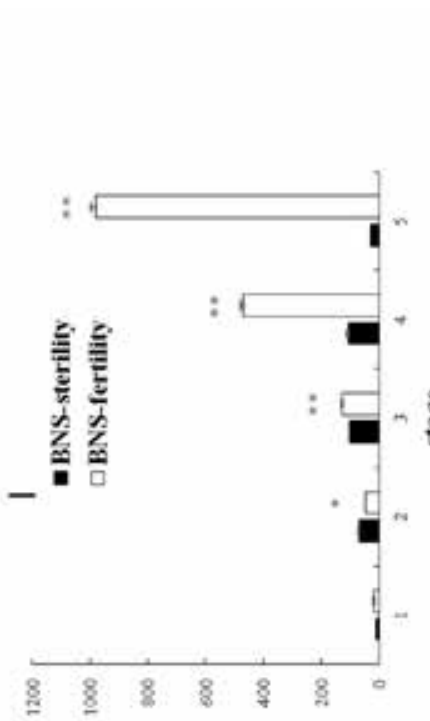

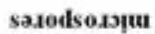
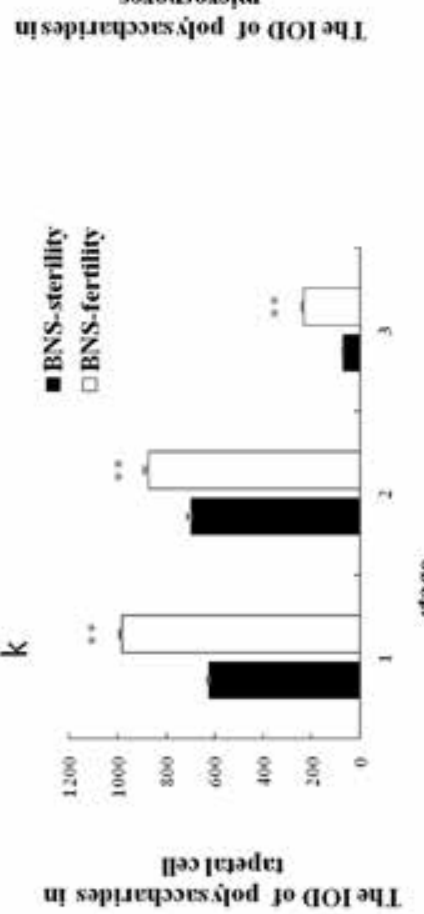

离 造

过

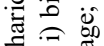

च

究

¿ $\frac{\pi}{5}$

咅 芯

.

司

㔄兽

충 $\frac{1}{6} 8$

创

$\leftarrow$ 造

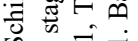

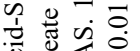

U⿺

寻合*

훙

히를 है

त छ

(c) की कै *

कें

Z

过勘员

称范

豞寻

으의

氖的

完苍范

(口)

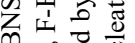

की

的要

Z桴

的吾

जि 要焉

क ज

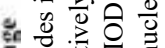

氜氙

के

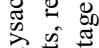

중 क्षे क

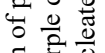

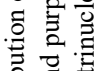

홀

E⿹

อ

항

声要

这告 

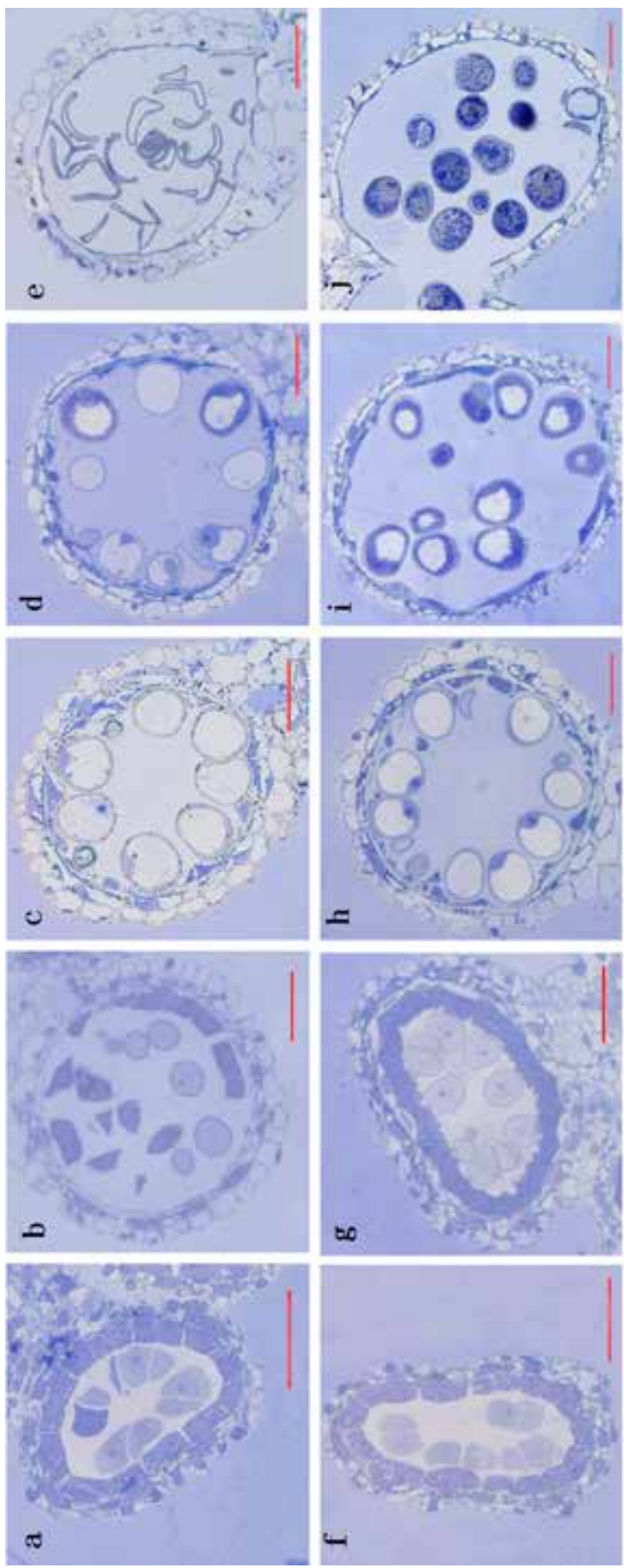

苍

言蓸

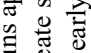

एक

究 ?

要要

in $=$ क

$\simeq$ e

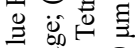

焉-18

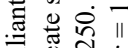

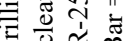

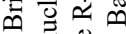

象节家

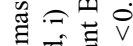

हैं

U

政

उ

ขั.

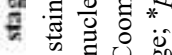

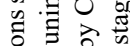

용

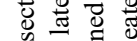

可产

进论主

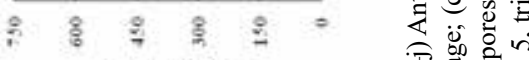

s.jodsenptu

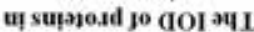

$\approx \tilde{\omega}$

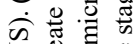

范

要

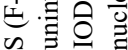

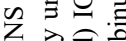

굴

至

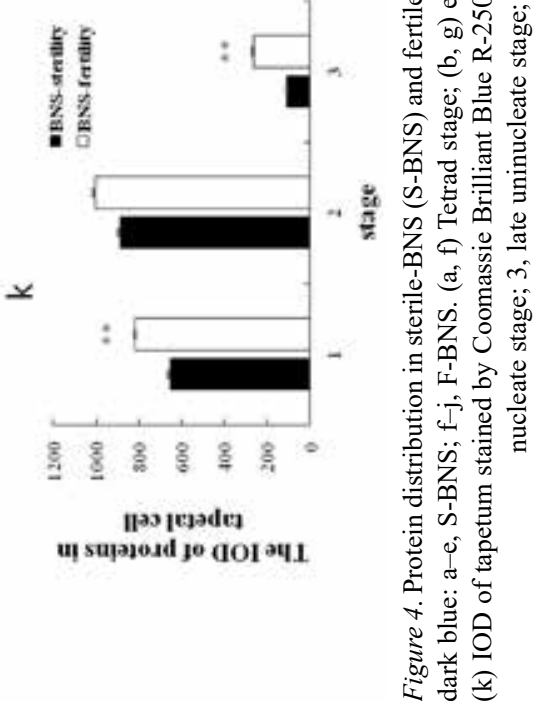

Cereal Research Communications 47, 2019 

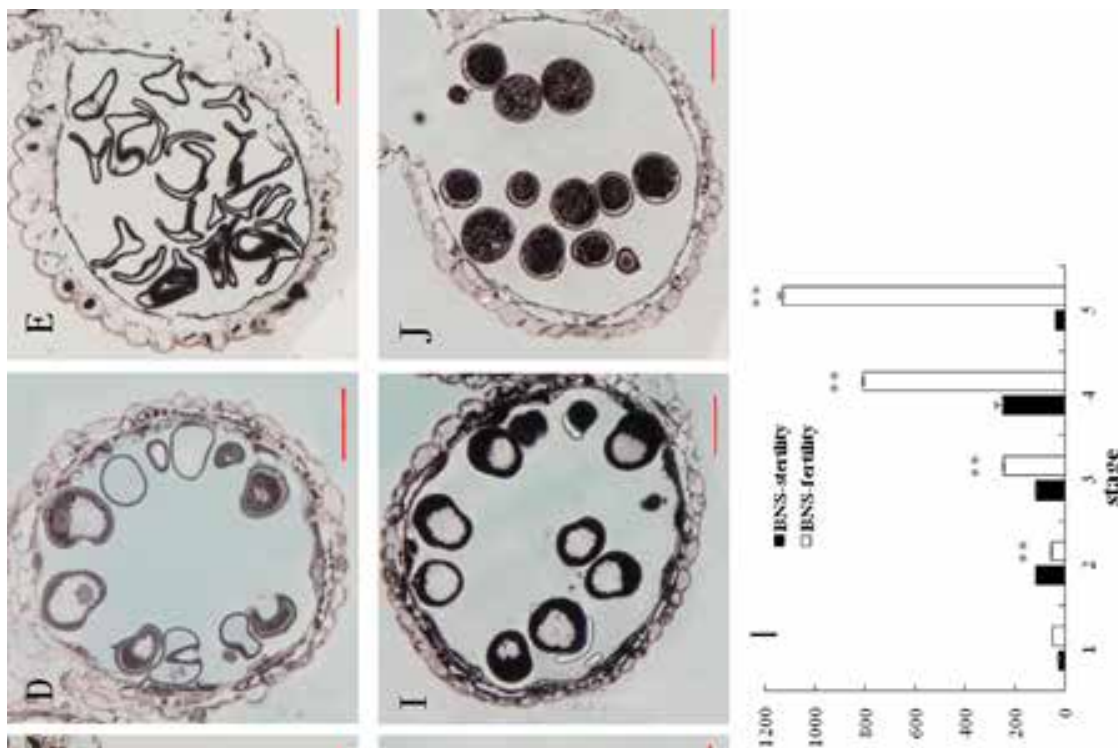

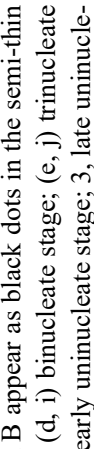
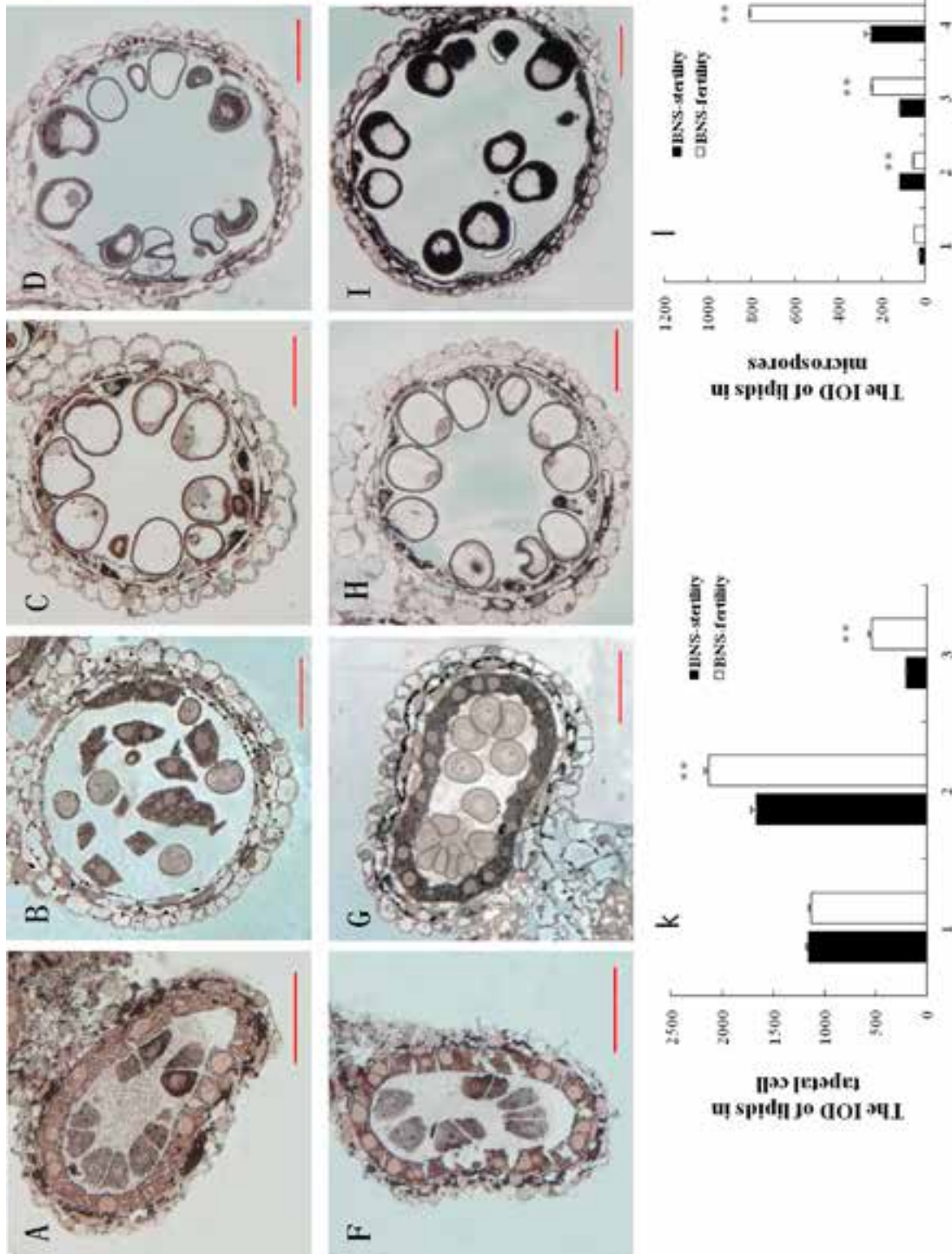

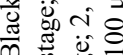

9 in 50

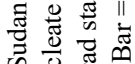

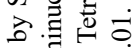

च $\cong 0$

㐘

का

㱐 $\frac{\pi}{0}$

i ज्ञ $\mathrm{V}$

플

$\exists$ 요

T

๘芑 ह

公录要

그늠

过 क्ष

त की की ?

ली

$0 \%$ क

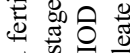

명

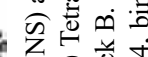

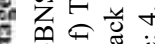

(2)

公完票

品

호

क्षे प्र

的焉

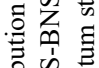

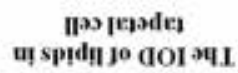

昰 i

可

흑 을

ni

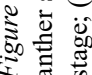


tapetum gradually detached and degraded. In both the F-BNS and S-BNS microspores, the IOD values increased from the tetrad stage (Figs $4 \mathrm{a}$ and $4 \mathrm{f}$ ) to the early uninucleate stage (Figs $4 \mathrm{~b}$ and $4 \mathrm{~g}$ ), and then decreased from the early uninucleate stage (Figs $4 \mathrm{~b}$ and $4 \mathrm{~g}$ ) to the late uninucleate stage (Figs $4 \mathrm{c}$ and $4 \mathrm{~h}$ ). No significant differences were identified between the IOD values for S-BNS and F-BNS tapetum in the early uniucleate stage, but the protein content of S-BNS microspores was significantly lower $(p<0.01)$ than that of F-BNS tapetum in the tetrad and late uninucleate stages (Fig. 4k). The protein content of F-BNS microspores exhibited an increasing trend from the tetrad stage to the trinucleate stage, whereas that of S-BNS microspores increased from the tetrad stage to the binucleate stage and then decreased significantly in the trinucleate stage (Fig. 4l). In the early uninucleate stage, the IOD values increased significantly for S-BNS microspores $(p<0.01)$ (Fig. 4l), and from the late uninucleate stage to the trinucleate stage, the IOD values were significantly lower for S-BNS microspores $(p<0.01)$ than those for F-BNS microspores (Fig. 41).

\section{Distribution and deposition of lipids in microspores}

The IOD values showed that in the tetrad stage, the lipid content of F-BNS tapetum was lower than that of S-BNS tapetum (Figs 5a, $5 \mathrm{f}$ and 5k), although the difference was not significant. From the early uninucleate stage to the late uninucleate stage, the IOD values were significantly higher $(p<0.01)$ for F-BNS tapetum (Figs $5 \mathrm{~g}$ and $5 \mathrm{~h}$ ) than those for S-BNS tapetum (Figs 5b and 5c). The lipid content of F-BNS microspores exhibited an increasing trend from the tetrad stage to the trinucleate stage, whereas the lipid content of S-BNS microspores increased from the tetrad stage to the binucleate stage and then decreased significantly in the trinucleate stage. Compared with F-BNS, the IOD values for S-BNS microspores decreased slightly in the tetrad stage, but this change was not significant. In the early uninucleate stage, the IOD values increased significantly $(p<0.01)$ for S-BNS microspores compared with those for F-BNS microspores (Fig. 51). From the late uninucleate stage to the trinucleate stage, the IOD values were significantly lower $(p<0.01)$ for S-BNS microspores than those for F-BNS microspores (Fig. 51).

\section{Discussion}

\section{Relationship between anther tapetum and microspore fertility}

The anther wall in monocots comprises the epidermis, inner wall, middle layer, and tapetum (Scott et al. 2004). Tapetal programmed cell death is a genetically controlled process, which is positively related to the vitality of microspores. PTC1 is known to be the key gene responsible for the regulation of tapetum development and pollen formation in rice (Li et al. 2011). This gene determines a switch for apoptosis and necrosis in tapetal cells, which is evident as delayed degradation and abnormal formation of the pollen wall. In wild rice, a mitochondrial DNA segment, Wild abortive 352 (WA352), encodes a 352-residue putative protein that interacts with a nuclear-encoded integral protein in the inner 
mitochondrial membrane, which may lead to cytoplasmic male sterility via tapetal programmed cell death (Luo et al. 2013). Li et al. (2006) studied cytoplasmic male sterility in onions and showed that tapetal programmed cell death occurs relatively early in sterile plants. Thus, sterility is probably associated with early degradation of the middle layer and tapetal cells.

The results of this study are in agreement with those of previous studies. S-BNS tapetum degraded in the early uninucleate stage, which was relatively earlier than that in F-SBN, and the degree of degradation was not consistent. The main cause of sterility in BNS might be the early degradation of tapetum hindering the normal development of microspores, thereby leading to pollen sterility.

\section{Relationship between nutrients and microspore sterility}

During the normal development process, microspores obtain nutrients from the tapetum to support their growth (Parish and Li 2010). The tapetum is a specialized cell layer between the sporogenous tissue and the anther wall, which functions as a source of nutrients for developing microspores, such as polysaccharides, lipids, and proteins (Wang et al. 2003). Insoluble polysaccharides are decomposed to intermediate metabolites via respiration to release the energy required for microspore development (Pacini 1996). Lipids provide protection against drying during sporopollenin precursor synthesis in the microspore wall (Wang et al. 2003). Proteins directly help microspores to maintain their metabolic and regulatory activities. The distribution and deposition of nutrients in anthers are important for normal microspore development.

In rice, the defective pollen wall (DPW) gene is expressed in both tapetal cells and microspores, and it participates mainly in primary fatty alcohol synthesis. The abnormal expression of DPW negatively affects the synthesis of structural substances in pollen grains and leads to pollen abortion (Shi et al. 2011). The pollen abortion mechanism responsible for physiological male sterility is related to the expression of the RAFTINI1 gene, which is relatively higher in sterile lines, and it stops sporopollenin secretion in the binucleate or trinucleate pollen stage, thereby leading to early tapetal cell death (Sheng et al. 2011). In a similar study, Liu et al. (2014) showed that the expression levels of proteins or enzymes, such as heat shock proteins, ATP enzyme subunits, glycosyltransferases, ubiquitin-proteasome, and $28 \mathrm{~S}$ protein enzyme subunits, differed significantly between sterile and fertile lines. Carbon starved anther (CSA), a rice mutant, encodes an R2R3 MYB domain protein that is mainly expressed in tapetal cells and that regulates sugar partitioning, which is necessary for rice pollen development. CSA leads to increases in the sugar contents of leaves and stems but decreases sugar content? in flowers with male sterility (Zhang et al. 2010).

In the present study, we found that large amounts of insoluble polysaccharides, lipids, and proteins were deposited in the nutritive cells in F-BNS until the trinucleate pollen stage. In the binucleate stage, the vacuoles disappeared and pollen inclusion increased gradually. In the trinucleate stage, these nutrients would help pollen grains mature and participate in fertilization normally. In S-BNS, the early degradation of microspores led 
to abortion of the nutritive and structural substances that are essential for the growth of microspores. Thus, the insoluble polysaccharide, lipid, and protein contents of pollen were limited after the binucleate pollen stage, thereby leading to pollen abortion. The results of this study are in agreement with those obtained by Li et al. (2013) who also investigated male sterility and the thermo-photosensitivity of BNS lines. However, it is still unclear whether the abnormal metabolism of nutrients is the direct cause of pollen abortion in wheat, and thus further research is required.

\section{Acknowledgements}

This study was sponsored by the National SupportProgram ofChina(No.2015BAD27B01), the National Natural Science Foundation of China (No. 31371697), the Technological Innovation and Over Planning Projects of Shaanxi Province (No. 2014KTZB02-01-02), the Fundamental Research Funds of Northwest A\&F University (No. Z109021304), Shannxi Postdoctoral Foundation (2016BSHED113), and American Zhongying Tang Breeding Foundation of Northwest A\&F University. Shannxi Provence's Key Project of Research and Development Plan (General Program) (2019NY-003).

\section{References}

Ariizumi, T., Hatakeyama, K., Hinata, K., Inatsugi, R., Nishida, I., Sato, S., Kato, T., Tabata, S., Toriyama, K. 2004. Disruption of the novel plant protein NEF1 affects lipid accumulation in the plastids of the tapetum and exine formation of pollen, resulting in male sterility in Arabidopsis thaliana. Plant J. 39:170-181.

Ahern, K. 1998. Image-Pro Plus. Biotechnol Softw I. J. 15:10-15.

Datta, R., Chourey, P.S., Pring, D.R., Tang, H.V. 2001. Gene-expression analysis of sucrose-starch metabolism during pollen maturation in cytoplasmic male-sterile and fertile lines of sorghum. Sex. Plant Reprod. 14:123-134.

Endo, M., Tsuchiya, T., Hamada, K., Kawamura, S., Yano, K., Ohshima, M., Higashitani, A., Watanabe, M., Kawagishi-Kobayashi, M. 2009. High temperatures cause male sterility in rice plants with transcriptional alterations during pollen development. Plant Cell Physiol. 50:1911-1922.

Fu, Z., Yu, J., Cheng, X., Zong, X., Xu, J., Chen, M., Li, Z., Zhang, D., Liang, W. 2014. The rice basic helixloop-helix transcription factor TDR interacting protein2 is a central switch in early anther development. Plant Cell 26:1512-1524.

$\mathrm{Hu}, \mathrm{S}$., Xu, L. 1990. A cytochemical technique for demonstration of lipids, polysaccharides and protein bodies in thick resin sections. J. Integr. Plant Biol. 32:841-846.

Karim, M.M., Siddika, A., Tonu, N.N., Hossain, D.M., Meah, M.B., Kawanabe, T., Fujimoto, R., Okazaki, K. 2014. Production of high yield short duration Brassica napus by interspecific hybridization between $B$. oleracea and B. rapa, Breeding Sci. 63:495-502.

Li, D., Li, G., Feng, S., Ru, Z. 2013. Cytochemical observation of fertile and sterile anthers of thermo-sensitive genic male-sterile wheat. Acta Agron. Sin. 39:878-884.

Li, H., Yuan, Z., Vizcay-Barrena, G., Yang, C., Liang, W., Zong, J., Wilson, Z., Zhang, D. 2011. Persistent tapetal cell1 encodes a PHD-finger protein that is required for tapetal cell death and pollen development in rice. Plant Physiol. 156:615-630.

Li, L., Ru, Z., Gao, Q., Jiang, H., Guo, F., Wu, S., Sun, Z. 2009. Male sterility and thermo-photosensitivity characteristics of BNS in wheat. Sci. Agric. Sin. 42:3019-3027.

Li, Y., Yang, Q., Yan, J., Chen, M. 2006. Cytoplasmic male-sterility of onion (Allium cepa L.) 63A is associated with premature degeneration of the Tapetum. Acta Agron. Sin. 32:369-372. 
Liu, H., Zhang, G., Zhu, W., Wu, W.K.K., Ba, Q., Zhang, L., Zhang, L., Niu, N., Ma, S., Wang, J. 2014. Differential proteomic analysis of polyubiquitin-related proteins in chemical hybridization agent-induced wheat (Triticum aestivum L.) male sterility. Acta Physiol. Plant 36:1473-1489.

Luo, D., Xu, H., Liu, Z., Guo, J., Li, H., Chen, L., Fang, C., Zhang, Q., Bai, M., Yao, N., Wu, H., Wu, H., Ji, C., Zheng, H., Chen, Y., Ye, S., Li, X., Zhao, X., Li, R., Liu, Y. 2013. A detrimental mitochondrial-nuclear interaction causes cytoplasmic male sterility in rice. Nat. Genet. 45:573-577.

Pacini, E. 1996. Types and meaning of pollen carbohydrate reserves. Sex. Plant Reprod. 9:362-366.

Parish, R., Li, S. 2010. Death of a tapetum: A programme of developmental altruism. Plant Sci. 178:73-89.

Regan, S., Moffatt, B. 1990. Cytochemical analysis of pollen development in wild-type Arabidopsis and a male-sterile mutant. Plant Cell 2:877-889.

Ru, Z., Zhang, L., Hu, T. 2015. Genetic analysis and chromosome mapping of the thermo-sensitive genic male sterile gene in wheat. Euphytica 201:321-328.

Scott, R.J., Spielman, M., Dickinson, H.G. 2004. Stamen structure and function. Plant Cell 16:546-560.

Sheng, Y., Zhang, G., Li, Y., Zhang, L., Wang, S., Zhao, X., Wang, L., Song, Y. 2011. The relationship on anther tapetum, sporopollenin and expression of RAFTIN1 in physiological male sterile wheat. Sci. Agric. Sin. 44:3937-3944.

Shi, J., Tan, H., Yu, X., Liu, Y., Liang, W., Ranathunge, K., Franke, R.B., Schreiber, L., Wang, Y., Kai, G., Shanklin, J., Ma, H., Zhang, D. 2011. Defective pollen wall is required for anther and microspore development in rice and encodes a fatty acyl carrier protein reductase. Plant Cell 23:2225-2246.

Wang, A., Xia, Q., Xie, W., Datla, R., Selvaraj, G. 2003. The classical Ubisch bodies carry a sporophytically produced structural protein (RAFTIN) that is essential for pollen development. Proc. Natl. Acad. Sci. USA 100:14487-14492.

Xie, C., Yang, Y., Qiu, Y., Zhu, X., Tian, H. 2005. Cytochemical investigation of genic male-sterility in Chinese cabbage. Sex. Plant Reprod. 18:75-80.

Zhang, D., Yang, L. 2014. Specification of tapetum and microsporocyte cells within the anther. Curr. Opin. Plant Biol. 17:49-55.

Zhang, B., Zhang, W., Gao, Q., Wang, M., Li, N., Zhang, Y., Wang, H., Gao, J., Zhao, L., Ru, Z. 2013. Genetic analysis on male sterility of thermo-photo-sensitive male sterile line BNS in wheat. Sci. Agric. Sin. 46:1533-1542.

Zhang, H., Liang, W., Yang, X., Luo, X., Jiang, N., Ma, H., Zhang, D. 2010. Carbon starved anther encodes a MYB domain protein that regulates sugar partitioning required for rice pollen development. Plant Cell 22:672-689.

Zhang, Z., Hu, T., Feng, S., Li, X., Li, G., Ru, Z. 2010. A preliminary study on fertility alteration of thermosensitive genic male sterile wheat line BNS. J. Henan Agri. Sci. 7:5-9. 